\title{
Optimization design of reconfiguration algorithm for high voltage power distribution network based on ant colony algorithm
}

https://doi.org/10.1515/phys-2018-0130

Received October 9, 2018; accepted November 14, 2018

Abstract: Distribution network reconfiguration is a very complex and large-scale combinatorial optimization problem. In network reconfiguration, whether an effective solution can be obtained is a key issue. Aiming at the problems in network reconstruction by traditional algorithm, such as long time required, more times of power flow calculation and high network loss, a network optimization design algorithm based on improved ant colony algorithm for high voltage power distribution network is proposed. After analyzing the operating characteristics of the high voltage power distribution network, the network topology of the high voltage power distribution network is described by constructing a hierarchical variable-structure distribution network model. A mathematical model of distribution network reconstruction considering the opportunity constraint with the minimum network loss as the objective function is established. The power flow distribution is calculated by using the pre-push back-generation method combined with the hierarchical structure of the distribution network. The maximum and minimum ant colony algorithm is introduced to improve the pheromone updating method of the traditional ant colony algorithm, and the search range is expanded, so that the algorithm can jump out of the local optimization trap to realize the accurate solution of the power distribution network reconstruction model. The experimental results show that compared with the current network reconstruction algorithm, the proposed algorithm requires less time for convergence, less power flow calculation, and lower network loss.

Keywords: Ant colony algorithm, high voltage power distribution network, network reconstruction, optimization design

PACS: $07.05 . \mathrm{Rm}, 84.70 .+\mathrm{p}$, 89.20.Ff

\footnotetext{
${ }^{\star}$ Corresponding Author: Yao Zhang: School of Accounting, Nanjing University of Finance and Economics, Nanjing China, E-mail: bzywbjb@hotmail.com
}

\section{Introduction}

As a relatively weak link in the power system, distribution networks are receiving increasing attention [1-4]. The state has planned to invest in the transformation of the old city network, and accordingly build a safe, reliable and flexible power distribution management system [5-9]. The core of the distribution management system is distribution automation. The distribution automation system is a comprehensive automation system consisting of a $10 \mathrm{kV}$ part of a $110 / 10 \mathrm{kV}$ substation (there is $66 / 10 \mathrm{kV}$ substation in some areas, and $35 / 10 \mathrm{kV}$ substation in rural areas), a $10 \mathrm{kV}$ feeder and all distribution system components from the $10 \mathrm{kV}$ feeder to the customer. Its purpose is to improve the reliability of power supply and achieve safe, economical and high-quality power supply. Network reconfiguration is an important part of distribution automation and an important software for urban and rural power grid reconstruction projects [10-13]. Reducing the line loss of the distribution network and improving the reliability of the power supply of the distribution network have always been the direction of the power companies. The line loss rate of western industrial countries is roughly 5\%-8\%, and that of China is about $9 \%$. Still, there is a gap compared with developed countries. Distribution network line loss of $35 \mathrm{kV}-110 \mathrm{kV}$ is an important part of regional line loss. In 1995, the distribution network line loss below 110 $\mathrm{kV}$ in national railway network accounted for $60 \%$ of the bus loss. It can be seen that reducing the line loss of the distribution network is one of the key issues in the loss reduction work [4]. In addition, according to incomplete statistics, $80 \%$ of user power failures are caused by power distribution system failures, and network reconstruction

\footnotetext{
Zhen Li: Zhongshan Institute, University of Electronic Science and Technology of China, Zhongshan, China, E-mail: lizhen123125136@163.com

Muhammad Aqeel Ashraf: International Water, Air \& Soil Conservation Society INWASCON, 59200, Kuala Lumpur, Malaysia, E-mail: ashraf@inwascon.org.my
} 
is an effective means to reduce distribution line losses and improve power supply reliability of distribution networks. The distribution system has the closest connection with the users, and the impact on the user's power supply reliability and power quality is also the most direct. A good distribution network topology not only saves a lot of investment and operating expenses for the power company, but also can improve the user's power supply satisfaction. Today, when the competition mechanism is continuously introduced into the power market, it is of great significance to study the power distribution network reconstruction algorithm [3].

Peng et al. applied the improved bacterial foraging algorithm to solve the problem of network reconstruction in distribution network. The group seeking mechanism of particle swarm optimization algorithm was introduced into the bacterial foraging algorithm. According to the defect of the bacterial foraging algorithm, the method of uniform variation propagation and the new dispersal rule were proposed. This method not only gives the guidance of algorithm search, but also protects the diversity of population after breeding, and effectively improves the algorithm's optimization speed and global search ability [9]. Deng et al. proposed a distribution network reconstruction algorithm based on improved artificial bee colony algorithm. In the improved algorithm, the neighborhood search method in the artificial bee colony algorithm was improved by the idea of differential evolution operation. The multi-dimensional search was performed directly in the discrete domain instead of the original single-dimensional update strategy, which improved the exploration ability of the algorithm and the convergence speed. In addition, in order to overcome the deficiencies based on proportional fitness selection, the selection probability based on fitness ranking was substituted for the selection probability based on proportional fitness, so that the diversity of the population was protected and the local optimality was avoided [2]. Zhu et al. proposed a distribution network reconstruction algorithm based on double-layer improved particle swarm optimization. In the process of switch combination optimization, in order to avoid the "Combined explosion" problem, the actual branch of the distribution network was divided into the branch set, and 0/1 coding was performed, which shortened the coding dimension and reduced the generation of infeasible solutions. The outer-layer improved particle swarm optimization algorithm was used to optimize the branch set combination, and the disconnection and closure of the branch set were determined according to the Sigmoid number; an inner-layer improved particle swarm optimization algorithm was proposed to optimize the ac- tual branch in the disconnected branch set, and the actual disconnected branch in the set was determined by the comparison method. The addition of distributed power in the network reconstruction reduced the network loss and improved the supporting for power voltage of node [1420].

Aiming at the problems of long time convergence, more times of power flow calculations and high network loss in distribution network reconstruction by using the current algorithm, the concept of ant colony algorithm is proposed and applied to the normal planning and failure recovery reconstruction of high voltage power distribution network, in order to achieve a better practical effect. The main research works are as follows.

When analyzing the operation characteristics of the distribution network, the topological structure of the distribution network is analyzed in detail, the simplified model processing method of the distribution network is given, and the layer-variable structure distribution network model is constructed to improve structural analysis efficiency in large-scale distribution network reconfiguration.

The objective function and constraint conditions of distribution network reconfiguration and the power flow calculation model of distribution network are given. The calculation method of pre-pushback power flow is introduced, and the improve pre-pushback power flow calculation method based on hierarchical operation is proposed.

By analyzing the defects of the traditional ant colony algorithm, the maximum and minimum ant colony algorithm (MMAS) is introduced in the optimal reconstruction of the distribution network. The pheromone update method combining local pheromone update and global pheromone update is established, and the range of pheromone is limited. The specific method of obtaining upper and lower limits is given.

\section{Methods}

\subsection{Network topology analysis and power flow calculation}

\subsubsection{Distribution network topology analysis}

The feeder of the distribution network is regarded as an undirected edge, which is described by $D$ matrix. The $D$ matrix is called a network base structure matrix, and its form is $n$ rows and $n$ columns, where $n$ is the number of distribution network nodes, and the form of $D$ matrix is as 
follows:

$$
D=\left[\begin{array}{cccc}
d_{11} & d_{12} & \cdots & d_{1 n} \\
& \vdots & & \\
d_{n 1} & d_{n 2} & \cdots & d_{n n}
\end{array}\right]
$$

If node $i$ and $j$ are connected by one edge, then $d_{i j}=$ $d_{j i}=1$, and the rest are not connected, then it is 0 . The $D$ matrix of the network topology describes the possible connection mode of the distribution network from a certain aspect, which is determined by the erection structure of the distribution network, so the diagram thus constructed is called the network base diagram [11].

The feeder line of the distribution network is regarded as the directional edge, and the direction is set to the direction of the power flow on the line. It is described by a $C$ matrix, which is in the form of $n$ rows and $n$ columns. The form of the $C$ matrix is as follows:

$$
C=\left[\begin{array}{cccc}
c_{11} & c_{12} & \cdots & c_{1 n} \\
& \vdots & & \\
c_{n 1} & c_{n 2} & \cdots & c_{n n}
\end{array}\right]
$$

If node $i$ and $j$ are connected by one edge and node $i$ points to $j$, then $c_{i j}=1$, called $c_{j i}=0$, and the remaining elements in the $C$ matrix are 0 . The $C$ matrix describes the actual operation mode of the distribution network. Therefore, the map thus constructed is called a mesh map. In an open-loop distribution network, a node with an ingress degree greater than 1 is generally a tie switch node of the network.

The network base structure matrix $D$, the source point distribution matrix $M$ and the joint distribution matrix $B$ are stored in the database according to the structure of the distribution network line construction, and can be modified and supplemented as the distribution network structure changes. The values of the elements in the node state matrix $T$ are derived from the report information of the data acquisition devices installed at each switch, including current transformer TA, voltage transformer TV, feeder terminal unit FTU, transformer terminal unit TTU and so on, wherein the feeder terminal unit FTU further comprises an on-rod FTU, an on-column FTU, a ring network cabinet FTU, and an open-closed FTU, etc. The variable structure model of the distribution network is mainly used to describe its network frame structure and topology [7]. For a distribution network structure with $N$ nodes, the following network base adjacency list matrix can be given:

$$
D T=\left[\begin{array}{ccccc}
d_{11} & d_{12} & d_{13} & d_{14} & d_{15} \\
& \vdots & & & \\
d_{n 1} & d_{n 2} & d_{n 3} & d_{n 4} & d_{n 5}
\end{array}\right]
$$

Where $d_{i 1}$ represents the node type of node $i$. The following formula is used to set the $C T$ matrix to reflect the operation mode of current distribution network:

$$
C T=\left[\begin{array}{ccccc}
c_{11} & c_{12} & c_{13} & c_{14} & c_{15} \\
& \vdots & & & \\
c_{n 1} & c_{n 2} & c_{n 3} & c_{n 4} & c_{n 5}
\end{array}\right]
$$

Where $c_{i 1}$ indicates the state of the node $i, 1$ indicates closed, 2 indicates open, and cc is always 1 for the T node and the bus node. For line stubs, $c_{i 1}$ is always $0 . c_{i 2}$ and $c_{i 3}$ indicate the heading starting point number of the trend direction of the node $i . c_{i 4}$ and $c_{i 5}$ are non-busbar nodes; for busbar nodes, they are meaningless [5].

The dissipative network model of the distribution network describes the load distribution properties of the distribution network and gives its matrix form:

$$
S T=\left[\begin{array}{cccc}
\dot{s}_{11} & \dot{s}_{12} & \dot{s}_{13} & \dot{s}_{14} \\
\vdots & & \\
\dot{s}_{n 1} & \dot{s}_{n 2} & \dot{s}_{n 3} & \dot{s}_{n 4}
\end{array}\right]
$$

Where $\dot{s}_{i 1}$ represents the load complex power flowing through node $i$, and $\dot{s}_{i 2} \sim \dot{s}_{i 4}$ represents the load complex power of the edge of the corresponding node. The order of the elements $\dot{s}_{i 2} \sim \dot{s}_{i 4}$ of $S T$ is the same as the order of the corresponding edges of $d_{i 3} \sim d_{i 5}$ in $D T$.

The rated load adjacency list can be set to the following matrix form:

$$
E T=\left[\begin{array}{cccc}
e_{11} & e_{12} & e_{13} & e_{14} \\
\vdots & & \\
e_{n 1} & e_{n 2} & e_{n 3} & e_{n 4}
\end{array}\right]
$$

where $e_{i 1}$ is the rated load of node $i, e_{i 2} \sim e_{i 4}$ is the rated load of the edge with the corresponding node as the end point, and the order of elements in ET is the same as that of $S T$.

In the research of distribution network reconfiguration, when using the pre-pushback method to calculate the power flow of the distribution network, establishing a hierarchical model data structure for the distribution network can be beneficial to the calculation of the system. In general, grid-type distribution network networks with multiple power supply modes are often open-loop, so most of the distribution network includes radial operation, ring network operation, and grid network operation, etc., it can be seen as a forest consisting of a power point as the root node, a stub node and a tie switch as the end point. For these radial point networks, a hierarchical model can be used for structural analysis. 


\subsubsection{Power flow calculation of power distribution network}

The power flow calculation determines the electrical state of the entire network according to the structure and operating conditions of a given network, which are mainly the voltage amplitude and phase angle of each node, the power distribution and power loss in the network, and performs cross-border inspection to understand and evaluate the health of the power distribution system [8]. It is an important basis for the quantitative analysis of the rationality, reliability and economy of the planning and design of the distribution system. In the distribution network reconfiguration, the network loss must be obtained through the power flow calculation, and the objective function is judged.

According to the above characteristics of the distribution network, the following assumptions are made: the network structure of the three-phase radiation distribution network is balanced; the three-phase radiation distribution network can be analyzed with a single-phase model; the charging capacitance and leakage current of the line are ignored; the node compliance power is known and remains constant.

For a distribution network with $n$ nodes and $n-1$ branches, node $e_{i}$ is used to describe the $i$ th node, and nodes node $e_{i}$ and node $e_{j}$ are used to form the branch $b_{i j}$. Assuming that the trend direction is node $e_{i}$ to node $_{j}$, node $_{i}$ is the parent node and node $e_{j}$ is the child node. The current and impedance values of the branch $b_{i j}$ are $I_{i j}$ and $R_{i j}+j X_{i j}$; the load current of the node node $e_{j}$ is $I_{j}$, the voltage is $U_{j}$, the power is $S_{j}$; the load current of the node node $e_{i}$ is $I_{i}$, the voltage is $U_{i}$, and the power is $S_{i}$. The steps to calculate the trend by pushback are as follows:

(1) Data initialization. Network topology data and the load value of each node are input; the number of iterations $k$ is set to 0 , and the load node voltage $\dot{U}_{i}$ is given to the initial value $\dot{U}_{i}^{0}$, the power root node voltage is known, the phase angle is 0 , and the voltages of the remaining nodes are all rated.

(2) The branch current $\dot{I}_{i j}^{k}$ is solved by push forward, for the stub circuit, the branch current is given below:

$$
\dot{I}_{i j}^{k}=\dot{I}_{j}^{k}=\left(\frac{S_{j}}{\dot{U}_{j}^{k}}\right)
$$

(3) From the peripheral branch current, the branch current before the previous parent node can be pushed forward. This value is the sum of the load current of the last node plus the current of all its sub-branches, namely:

$$
\dot{I}_{i j}^{k}=\left(\frac{S_{j}}{\dot{U}_{j}^{k}}\right)+\sum_{l \in a(j)} \dot{I}_{j i}^{k}
$$

where $a(j)$ is the set of all the branches with node $j$ as the parent node.

(4) The node voltage $\dot{U}_{i}^{k}$ is obtained by pushback. Through the above steps, after obtaining all the branch currents $\dot{I}_{i j}^{k}$, the voltage values of the respective nodes can be obtained successively from the root node of the power source:

$$
\dot{U}_{j}^{k}=\dot{U}_{i}^{k}-\dot{I}_{i j}^{k} \times\left(R_{i j}+j X_{i j}\right)
$$

(5) The voltage amplitude correction value $\Delta \ddot{U}_{i}$ of each node is calculated:

$$
\Delta \ddot{U}_{i}=\left|\dot{U}_{j}^{k}-U_{j}^{k-1}\right|
$$

(6) The maximum value $\max \left(\Delta \dot{U}_{i}\right)$ of the node voltage correction value is calculated.

(7) To verify the convergence condition. When the condition $\max \left(\Delta \dot{U}_{i}\right)<\varepsilon$ is satisfied, $\varepsilon$ indicates the convergence criterion precision value, then the iteration converges at this time, and the power flow calculation result is obtained; otherwise, $k \leftarrow k+1$ goes to step (2) to continue execution.

In view of the fact that the distribution network is generally a closed loop design and open loop operation structure. Each node powered by a power point can be regarded as a tree structure with a power point as a root node. When the distribution network is in normal operation, it can be decomposed into multiple dendritic radiation network structures. In these structures, the nodes and branches are divided into several layers according to the direction of the power flow, the voltage and current are calculated in layers, and the hierarchical calculation of the branches or nodes can be performed in the same layer of different trees. For relatively large-scale radial networks, stratification can significantly increase the processing speed of power flow calculations [10].

For a high voltage power distribution network with $n$ nodes, after stratification operation, a network hierarchy matrix $L$, a branch-to-end node matrix $f$, a head node matrix $t$, and a branch-level association matrix $E$ may be formed. The specific steps are given below:

(1) Data initialization. The number of layers $i$ is the total number of layers of the distribution network, that is, $i=a$, while the array $Q$ is empty.

(2) It is judged whether the forward pushing process is ended by the value of $i$, and when the $i=0$ is satisfied, the forward pushing process ends, and when $i \neq 0$ is satisfied, the non-zero elements in the network hierarchical matrix $L$ are placed in $Q$. 
(3) It is judged whether the array $Q$ is empty; if it is empty, $i=i+1$, and the process goes to the step (2).

(4) An element is selected from $Q$ and it is set as $x$, and the elements in the branch hierarchy association matrix $E$ are searched.

If $x$ is not in all elements, the current of branch $x$ is:

$$
\dot{I}_{i j}^{k}=\dot{I}_{j}^{k}=\left(\frac{S_{j}}{\dot{U}_{j}^{k}}\right)
$$

Otherwise, $E_{y(1)}=E_{y(2)}=\cdots=E_{y(p)}=x$, then the current of branch $x$ is:

$$
\dot{I}_{i j}^{k}=\left(\frac{S_{j}}{\dot{U}_{j}^{k}}\right)+\sum_{l \in a(j)} \dot{I}_{j i}^{k}
$$

After obtaining the current of the branch $x$, return to step (3) and continue to calculate the next branch.

The pushback steps of layered voltage are:

(1) Initialization. The number of layers $x$ is 1 , that is, $\iota=1$, and the array $Q$ is set to an empty array.

(2) Determination of the value of $Q$. Assuming $\iota=\alpha+1$, the voltage pushback process ends (Fletcher et al. 2018); otherwise, the non-zero elements in the network hierarchy matrix $L$ are placed in $Q$.

(3) It is judged whether or not the array $Q$ is empty, and if it is empty, it returns to the step (2).

(4) An element is selected from $Q$, and set as $x$, and the sending end node matrix $f$ and the head end node matrix $t$ are searched, to obtain the sending end node $f_{x}$ and the receiving end node $t_{y}$ of the branch $x$, then the voltage of node $t_{y}$ is:

$$
\dot{U}_{t_{y}}^{k}=\dot{U}_{f_{x}}^{k}-\dot{I}_{m}^{k} \times\left(R_{x}+j X_{i j}\right)
$$

After obtaining the voltage of the node $t_{y}$, it goes to step (3) and continues to calculate the next node to be sought.

\subsection{PSTUA-based mathematical model for distribution network reconstruction}

The mathematical model of distribution network reconstruction based on PSTUA fully considers the uncertainty of the output of distributed wind turbines and photovoltaic power generation systems. It is modeled by probability and statistics method: the objective function is the minimum mathematical expectation of system network loss, which can be obtained by passing LHS MCS calculation. The constraints mainly consider the power flow equation constraint, the node voltage constraint, the branch power flow constraint and the network topology constraint. Wherein, the node voltage and the branch power flow constraint are given in the form of opportunistic constraints [6]. Based on the minimum mathematical expectation of system network loss, a mathematical model of distribution network reconstruction considering opportunity constraints is established, namely:

$$
\min P_{L}=E\left(\sum_{k=1}^{N_{L}} P_{k, L}\right) \approx \frac{1}{N_{L H S}} \sum_{i=1}^{N_{L H S}} \sum_{k=1}^{N_{L}} I_{i, k}^{2} r_{k}
$$

Where, $P_{L}$ represents the expectation value of system network loss, $E$ represents the mathematical expectation of calculating the random variable, $N_{L}$ represents the number of system branches, $P_{k, L}$ represents the random variable, $I_{i, k}^{2}$ represents the current flowing through the branch in the ith sampling, and $r_{k}$ represents the branch resistance.

The constraints of power flow equation are:

$$
\left\{\begin{array}{l}
P_{i, S}=V_{i} \sum_{j \in \Omega_{i}} V_{j}\left(G_{i j} \cos \theta_{i j}+B_{i j} \sin \theta_{i j}\right) \\
Q_{i, S}=V_{i} \sum_{j \in \Omega_{i}} V_{j}\left(G_{i j} \sin \theta_{i j}-B_{i j} \cos \theta_{i j}\right)
\end{array}\right.
$$

Wherein, $P_{i, S}$ and $Q_{i, S}$ respectively indicate that the active power and the reactive power are injected at the node $i$, $V_{i}$ and $V_{j}$ respectively represent the voltage amplitudes at the node $i$ and $j$, respectively, $\Omega_{i}$ represents the other node sets connected to the node $i$, and $G_{i j}$ and $B_{i j}$ respectively represent the conductance and susceptance of the branch $j, \theta_{i j}$ represents the phase angle difference between node $i$ and node $j$, and $N$ represents the total number of nodes.

The voltage constraint of node is:

$$
\operatorname{Pr}\left\{V_{i}^{\min } \leq V_{i} \leq V_{i}^{\max }\right\} \geq \beta_{U}
$$

Where $P_{r}$ represents the establishment probability of event, $V_{i}^{\max }$ and 3 respectively represent the upper and lower limits of the voltage amplitude at node $i, i \in \Theta$; where, $\Theta$ represents the system node set, and $\beta_{U}$ represents the confidence level of the voltage constraint.

The constraints of branch power flow are:

$$
\operatorname{Pr}\left\{\left|P_{j}\right| \leq P_{j}^{\max }\right\} \geq \beta_{I}
$$

Where, $P_{j}^{\max }$ indicates the upper limit of the active power of the $j$ th branch transmission, and $\beta_{I}$ indicates the confidence level of the constraints of branch power flow.

The topology constraints of high voltage power distribution network are:

$$
g \in G
$$

Where, $g$ represents the topology of current high voltage distribution network, and $G$ represents all network topology sets that satisfy the radial structure. 


\subsection{Solving network reconstruction model based on maximum and minimum ant colony algorithm}

The traditional ant colony algorithm is applied to the optimization and reconstruction of distribution network, which is easy to fall into the local optimal solution. In addition, the convergence speed of the above traditional ant colony algorithm needs to be improved. How to avoid prematurely falling into the local optimal solution and improve the convergence speed of the algorithm is the problem to be solved by the improved ant colony algorithm. The maximum-minimum ant colony algorithm (MMAs) solves both problems well.

The improvement of the traditional ant colony algorithm by MMAS is mainly reflected in the pheromone, mainly for the following three points:

(1) Setting the upper and lower limits of the pheromone. When the update of a pheromone causes the value of the tributary pheromone to be greater than the upper limit, the pheromone value is equal to the upper limit, and the value of the pheromone is less than the value of the pheromone due to the volatilization of the pheromone. The lower limit causes the pheromone value to be equal to the lower limit. (2) The initial pheromone concentration is set to a maximum value, and the pheromone volatilization coefficient is set to a smaller value;

(3) A pheromone update method combining local pheromone update method and global pheromone update method are adopted.

The above three points will be described in detail below.

In order to avoid the case where the ant colony algorithm converges to the local optimal solution due to pheromone accumulation, the MMAS forcibly limits the maximum and minimum values of the pheromone concentration, and makes $\tau_{\min }<\tau_{i^{\prime} j^{\prime}}(t)<\tau_{\max }$ for each path. After each iteration, it ensures that the value of the pheromone concentration is still within this range. When $\tau_{i^{\prime} j^{\prime}}(t)>\tau_{\max }$, let $\tau_{i^{\prime} j^{\prime}}(t)=\tau_{\max }$; when $\tau_{i^{\prime} j^{\prime}}(t)<\tau_{\max }$, let $\tau_{i^{\prime} j^{\prime}}(t)=\tau_{\min }$. Also it makes sure that $\tau_{\min }>0$, so that the probability that each path is selected is not zero.

Under the MMAS algorithm, the pheromone $\tau_{\max }$ is set to a progressive maximum limit. Each time, a new solution is obtained through the population iteration, $\tau_{\max }$ is updated to obtain a value that dynamically changes with the number of iterations (Zhou \& Littler, 2016).

In order to determine the value of $\tau_{\min }$, the following assumptions need to be made:

(1) The optimal solution will be searched before the search stagnation occurs. Under this assumption, the probability of finding the global optimal solution after the iteration is completed will be much greater than 0 , which will ensure that a better solution is found until the optimal solution is found.

(2) The ability to find the optimal solution depends mainly on the selection of the upper and lower limits of the pheromone, rather than the selection of the heuristic function.

The rationality of the first hypothesis is determined by the size of the search space, which means that there is a possibility of finding a better solution near the good solution. The second hypothesis is based on the following set of methods for setting the pheromone's minimum value of $\tau_{\min }$. Since the distribution network reconstruction takes $\eta_{i^{\prime} j^{\prime}}=1 / R_{i^{\prime} j^{\prime}}$, which is a constant, the influence of the heuristic function on the transition probability can be ignored. In the MMAS algorithm, the value of parameter $\beta$ is taken very small or simply no heuristic function is used.

Under the premise of the above assumptions, a relatively good value of $\tau_{\min }$ can be obtained in a progressive manner. When the MMAS algorithm has converged, the probability value of the resulting optimal solution is a value $P_{b}$ that is much larger than zero. In this case, the ant that got the optimal solution made a "correct" choice on each node, and the pheromone concentration on the selected path is a maximum of $\tau_{\max }$. In fact, at each node, the probability $P_{d}$ of selecting the optimal path accordingly depends directly on $\tau_{\max }$ and $\tau_{\min }$. For the sake of simplicity, assuming that $P_{d}$ is constant on each node facing the selection. An ant must make the $n^{\prime}$ times of correct choice and the probability of finding the optimal solution is $P_{d}^{n^{\prime}}$. Then,

$$
P_{d}^{n^{\prime}}=P_{b}
$$

which is:

$$
P_{d}=\sqrt[n^{\prime}]{P_{b}}
$$

Given a value of $P_{b}$, it can reasonably set the value of $\tau_{\min }$. On average, at each node, the ant needs to make a choice among the avg branches. Ignoring the heuristic function, the optimal path pheromone concentration is $\tau_{\max }$, and the pheromone concentration on other paths is $\tau_{\min }$ in the extreme case, then the branch transition probability of selecting the optimal path on each node is:

$$
P_{b}=\frac{\tau_{\max }}{\tau_{\max }+(\text { avg }-1) \tau_{\min }}
$$

The above equation is solved to get:

$$
\tau_{\min }=\frac{\tau_{\max }\left(1-P_{d}\right)}{(a v g-1) P_{d}}=\frac{\tau_{\max }\left(1-\sqrt[n^{\prime}]{P_{b}}\right)}{(a v g-1) \sqrt[n^{\prime}]{P_{b}}}
$$

Analysis of the above equation shows that if $P_{b}=1$ is satisfied, then $\tau_{\min }=0$. 
By the above formula, it is assumed that given a $P_{b}$, the value of $\tau_{\min }$ can be determined. When the MMAS converges, the selection of the value of $P_{b}$ is directly related to the number of solutions found by the algorithm. According to the statistical law, if the number of ants in the ant colony is large enough, the macroscopic manifestation of $P_{b}$ is the proportion of the ant that obtains the optimal solution in a certain iteration to the total ants in the ant colony [1]. Generally, it is set as:

$$
\tau_{\min }=\frac{\tau_{\max }}{2 n^{\prime}}
$$

Where $n^{\prime}$ indicates the number of system nodes.

In order to speed up the convergence of the algorithm, it is hoped that the pheromone of all branches will be updated to $\tau_{\max }(1)$ after the first iteration is completed. By arbitrarily setting a relatively large initial value of $\tau(0)$, after the first iteration of the MMAS, the value of the pheromone is forcibly limited to the given range. In general, the setting is:

$$
\tau(0)=\tau_{\max }(1)
$$

In the first few iterations, choosing this pheromone initialization method will speed up the convergence of the algorithm.

Different from the traditional ant colony algorithm, MMAS adopts the method of local pheromone update and global pheromone update. The local pheromone update method is the pheromone update after the completion of an ant traversal in the iteration. The global pheromone update is a pheromone update to the global after an iteration is completed. The two pheromone update methods are introduced as follows:

(1) Local update method of pheromone

Supposing when the program proceeds to the DD iteration, after the $\kappa$ th ant traversal is completed, if the ant-derived network is subjected to load flow calculation constraints, and the network loss of the network is smaller than the current optimal solution, then the selected branch pheromone by this ant will be updated [12]. The update formula is as follows:

$$
\begin{gathered}
\tau_{i^{\prime} j^{\prime}}^{\kappa}=\left(1-\rho \cdot \tau_{i^{\prime} j^{\prime}}^{\kappa-1}\right) D\left(D+\Delta \tau_{i^{\prime} j^{\prime}}^{\kappa} D D\right) \\
\Delta \tau_{i^{\prime} j^{\prime}}^{\kappa} D D=Q / p s_{\kappa}
\end{gathered}
$$

Where $i^{\prime} j^{\prime}$ denotes a branch $i^{\prime} j^{\prime}, \rho$ denotes a pheromone's volatilization coefficient $(0 \leq \rho<1), \kappa$ denotes the number of ants, $\Delta \tau_{i^{\prime} j^{\prime}}^{\kappa} D D$ denotes a change amount of pheromone on the branch $i^{\prime} j$, and 8 denotes a local pheromone's update parameter, that is, $Q=\tau_{0}, p s_{\kappa}$ is the $\kappa$ th ant traversing the network loss of the resulting network.

(2) Global update method of pheromone
When the program proceeds to the $D D$ th iteration and is ready for the DD+1th iteration, if the network of this iteration is better than the current optimal solution by the power flow calculation, the pheromone of all the branches will be updated once. The update formula is as follows:

$$
\begin{gathered}
\tau_{i^{\prime} j^{\prime}}(D D+1)=\left(1-\rho \cdot \tau_{i^{\prime} j^{\prime}}\right) D(D+1) \Delta \tau_{i^{\prime} j^{\prime}} D D \\
\Delta \tau_{i^{\prime} j^{\prime}} D D=\sum_{\kappa=1}^{m} \Delta \tau_{i^{\prime} j^{\prime}} D L \\
\Delta \tau_{i^{\prime} j^{\prime}}^{\kappa}(D D)=Q^{\prime} / p s_{\kappa}
\end{gathered}
$$

In the traditional ant colony algorithm, each tributary is updated with pheromone after the iteration is completed, and after the iteration is completed in the MMAS, only the tributary pheromone on the optimal network is updated. The revised pheromone update rules are given below:

$$
\tau_{i^{\prime j^{\prime}}}(D D+1)=(1-\rho) \tau_{i^{\prime} j^{\prime}}(D D)+\Delta \tau_{i^{\prime}, j^{\prime}}^{b}(D D)
$$

Where, $\Delta \tau_{i^{\prime}, j^{\prime}}^{b}(D D)=Q^{\prime} / f\left(s_{b}\right)$ represents the objective function value of the optimal solution $s_{b}$ found in the last iteration or the previous solution $3 s_{g b}$, and obviously, in the high-voltage distribution network reconstruction, there are:

$$
f\left(s_{b}\right)=p s_{b}
$$

Combining with the pheromone update method, the disadvantages of the traditional ant colony algorithm are analyzed. The idea of using the maximum and minimum ant colony algorithm for distribution network reconfiguration is put forward. Finally, the detailed description and analysis of the pheromone setting method are given and the pheromone update method of the traditional ant colony algorithm is improved.

\section{Results}

In order to objectively evaluate the reconstruction performance of each algorithm, a unified reconstruction computing platform is established for each algorithm. The four algorithms applied have different heuristic rules for the main part only,and the other modules are completely identical. The following modules are mainly unified:

(1) Reconstruct the mathematical model. All algorithms construct an objective function with reducing the network active power loss as the optimization target, and the mathematical model described in Section 2.2 is used. 
(2) Distribution network power flow calculation. The layered model approach is introduced, and a layered forward pushback push flow calculation method is proposed.

(3) Loop search of network.

(4) MATLAB software is used to make programming and testing. All algorithms use MATLAB R2014a to prepare the distribution network reconfiguration program. The PC model for testing and analysis is Inter(R) Core(TM) i32310M CPU@2.10GHz and memory is 4.00GB.

The effectiveness, accuracy and versatility of each algorithm for solving the distribution network reconstruction problem are tested by using three classical test systems and three large real networks as examples.

(1) Reconstruction analysis of improved bacterial foraging algorithm

The improved bacterial foraging algorithm is applied to solve the network reconstruction problem of high voltage distribution network. Table 1 shows the results of reconstruction of six test systems based on the improved bacterial foraging algorithm.

It can be seen from the results given in Table 1 that the Taipower84, PG\&E69, and Bus119 test systems can obtain the optimal solution. The IEEE33 and Taipower84 node systems do not obtain the optimal solution, and the obtained optimal solution has little difference from the global optimal solution. According to the reconstruction results given in Table 1, the improved bacterial foraging algorithm can effectively solve the network reconstruction problem of high voltage power distribution network.

(2) Reconstruction analysis of the proposed algorithm

The proposed algorithm is used to solve the problem of power distribution network reconstruction. Table 2 lists the reconstruction results of multiple examples based on ant colony algorithm, and the reconstruction results of the same example are given by the optimal flow pattern algorithm.

It can be seen from Table 2 that applying the optimal flow pattern algorithm to solve the distribution network reconstruction can obtain a feasible radial network structure, but only the IEEE16 system obtains the optimal solution, and other systems are trapped in local optimum, and the optimization performance is poor. Thus, the final solution of the optimal flow pattern algorithm is used as the initial solution of the proposed algorithm for reconstruction calculation. It can be seen from Table 2 that in the reconstruction results based on the ant colony algorithm, the IEEE16, IEEE33, PG\&E69, 119 nodes and 136-node systems all converge to obtain the global optimal solution, and only the Bus119 system does not find the optimal solution. Comparing Tables 1 and 2 shows that the improved bacterial foraging algorithm and the proposed algorithm can be used to solve the problem of distribution network reconstruction. The proposed algorithm has better optimization performance.

In order to comprehensively compare the reconstruction performance of the improved bacterial foraging algorithm and the applied ant colony algorithm, Table 3 lists the time required for the two algorithms to converge on the same test system, the number of power flow calculations, and the lowest value of system node voltage. From the data in Table 3, it can be concluded that applying the improved bacterial foraging algorithm needs to calculate more network flows than the application of the ant colony algorithm for distribution network reconstruction, and the time required is more than that of applying the ant colony algorithm. The ant colony algorithm performs convergence faster in distribution network reconstruction. For the six test systems, only the Bus119 system cannot achieve the optimal solution in network reconstruction based on ant colony algorithm, and the other can obtain the optimal solution. The effective of improved bacterial foraging algorithm for large-scale network reconstruction is generally satisfied and is easy to fall into the local optimal. Table 3 also shows the minimum value of the node voltage in the network after the test system uses the improved bacterial foraging algorithm and the ant colony algorithm.

In summary, applying the improved bacterial foraging algorithm and the ant colony algorithm can solve the problem of distribution network reconstruction effectively, quickly and accurately. The ant colony algorithm has better performance than the improved bacterial foraging algorithm. It is suitable for solving large-scale network reconfiguration calculations, both online and offline.

(3) Comparison of performance of multiple reconstruction algorithms

Taking the IEEE33 node system and the TaiPower84 node system as examples, the four reconstruction algorithms of the actual application are compared with the performance of the reconstruction calculation time, power flow calculation times, final network loss and node voltage minimum value; Part of the algorithms with excellent performance under the single indicator are selected to combine with other indicators for further comprehensive analysis.

The IEEE33 node system has 36 branches and 8 tie switches with a rated voltage of $13.52 \mathrm{kV}$ and a total load of $3.712 \mathrm{MW}+\mathrm{j} 2.5 \mathrm{Mvar}$. The node voltage has a minimum value of $0.8542 \mathrm{pu}$.

The TaiPower84 node system has a total of 95 branches and 12 switching switches. The rated switch is $12.5 \mathrm{kV}$ and the total load is $28.25 \mathrm{MW}+\mathrm{j} 21.5 \mathrm{Mvar}$. 
Table 1: Reconstruction results based on improved bacterial foraging algorithm

\begin{tabular}{lllll}
\hline Test system & Initial solution & Initial network loss / kW & Improved bacterial foraging algorithm \\
& & & Final solution & Final network loss / kW \\
\hline IEEE16 & $\{16,20,25\}$ & 510.22 & $\{18,15,24\}$ & 475.52 \\
IEEE33 & $\{32,33,34,35,36\}$ & 201.25 & $\{7.9,11,35,30\}$ & 138.54 \\
PG\&E69 & $\{69,70,71,72,73\}$ & 224.00 & $\{69,70,71,72,73\}$ & 98.52 \\
Taipower84 & $\{83,84,85,86,87,88\}$ & 530.25 & $\{83,84,85,86,87,88\}$ & 472.65 \\
Bus119 & $\{118,119,120,121,122,123\}$ & 1285.25 & $\{118,119,120,121,122,123\}$ & 875.55 \\
Bus136 & $\{118,119,120,130,131,132\}$ & 319.25 & $\{136,168,140,142,144,146\}$ & 295.54 \\
\hline
\end{tabular}

Table 2: Reconstruction results based on ant colony algorithm

\begin{tabular}{|c|c|c|c|c|}
\hline \multirow{2}{*}{ Test system } & \multicolumn{2}{|c|}{ Optimal flow pattern algorithm } & \multicolumn{2}{|l|}{ The proposed algorithm } \\
\hline & Optimal solution & $\begin{array}{l}\text { Final network loss } \\
/ \mathrm{kW}\end{array}$ & Optimal solution & $\begin{array}{l}\text { Final network loss } \\
/ \mathrm{kW}\end{array}$ \\
\hline IEEE16 & $\{18,16,24\}$ & 455.58 & $\{18,16,24\}$ & 455.58 \\
\hline IEEE33 & $\{8,12,16,36,34\}$ & 142.25 & $\{8,12,16,36,34\}$ & 138.54 \\
\hline PG\&E69 & $\{16,12,53,12,58\}$ & 108.36 & $\{68,70,54,12,60\}$ & 106.25 \\
\hline Taipower84 & $\{32,42,88,86,38,62\}$ & 472.02 & $\{32,42,88,86,38,62\}$ & 472.65 \\
\hline Bus119 & $\{74,20,70,42,50,24\}$ & 884.25 & $\{24,22,38,34,42,50\}$ & 868.25 \\
\hline Bus136 & $\{10,52,46,146,118,68,146\}$ & 280.54 & $\{142,145,135,146,132,121,148\}$ & 280.54 \\
\hline
\end{tabular}

Table 3: Comparison of reconstruction performance of different algorithms

\begin{tabular}{lllllllll}
\hline Test system & $\begin{array}{l}\text { The improved bacterial foraging } \\
\text { algorithm } \\
\text { Number } \\
\text { of power } \\
\text { flows }\end{array}$ & time/s & Vmin/pu & $\begin{array}{l}\text { Is it } \\
\text { optimal? }\end{array}$ & $\begin{array}{l}\text { the ant colony algorithm } \\
\text { Number } \\
\text { of power } \\
\text { flows }\end{array}$ & time/s & Vmin/pu & $\begin{array}{l}\text { Is it } \\
\text { optimal? }\end{array}$ \\
\hline IEEE16 & 6 & 0.124 & 0.9725 & Yes & 5 & 0.045 & 0.9725 & Yes \\
IEEE33 & 20 & 0.675 & 0.9245 & Yes & 10 & 0.185 & 0.9245 & Yes \\
PG\&E69 & 12 & 1.352 & 0.9314 & Yes & 11 & 0.310 & 0.9314 & Yes \\
Taipower84 & 26 & 3.254 & 0.9415 & Yes & 23 & 0.482 & 0.9415 & Yes \\
Bus119 & 32 & 8.845 & 0.9335 & No & 30 & 0.965 & 0.9235 & No \\
Bus136 & 22 & 11.254 & 0.9425 & No & 20 & 0.720 & 0.9425 & Yes \\
\hline
\end{tabular}

In order to compare the computational efficiency of the four algorithms in practical application, Figure 1 shows the time spent by the IEEE33 system on the reconstruction of each algorithm based on the radar graph. It can be seen from Figure 1 that the proposed reconstruction algorithm has the fastest calculation speed, and the distribution network reconstruction algorithm based on the improved bacterial foraging algorithm is the second. The calculation algorithm based on the improved artificial bee colony algorithm is the third. The distribution network reconstruction algorithm based on the double-layer improved particle swarm optimization algorithm takes the longest time. In order to further verify the versatility and accuracy of the algorithms, Figure 2 shows the calculation time of the TaiPower84 node system reconstruction. Similarly, the running speed of proposed reconstruction algorithm is also the fastest, followed by the improved bacterial foraging algorithm and the improved artificial ant colony algorithm, while the longest calculation time is based on the double-layer improved particle swarm optimization algorithm. In order to simplify the description, the proposed algorithm, the improved bacterial foraging algorithm, the improved artificial ant colony algorithm, and double-layer improved particle swarm optimization algorithm are described as ACO, BFOA, ABC, PSO, respectively.

For the distribution network reconstruction, the power flow calculation runs through the algorithm. Efficient power flow calculation methods can reduce the number of power flow calculations and improve the reconstruction efficiency of algorithm. For the IEEE33 and TaiPower84 systems, Table 4 shows the number of power flow calculations required for the reconstruction by the four algorithms.

Analysis of the data in Table 4 shows that the algorithm for the IEEE33 node system calculates the power flow 
Table 4: The number of power flow calculations required for the reconstruction by the four algorithms in IEEE33 and TaiPower84 systems

\begin{tabular}{lll}
\hline Algorithm & IEEE33 system & TaiPower84 system \\
\hline ACO & 10 times & 20 times \\
BFOA & 22 times & 26 times \\
ABC & 22 times & 28 times \\
PSO & 32 times & 75 times \\
\hline
\end{tabular}

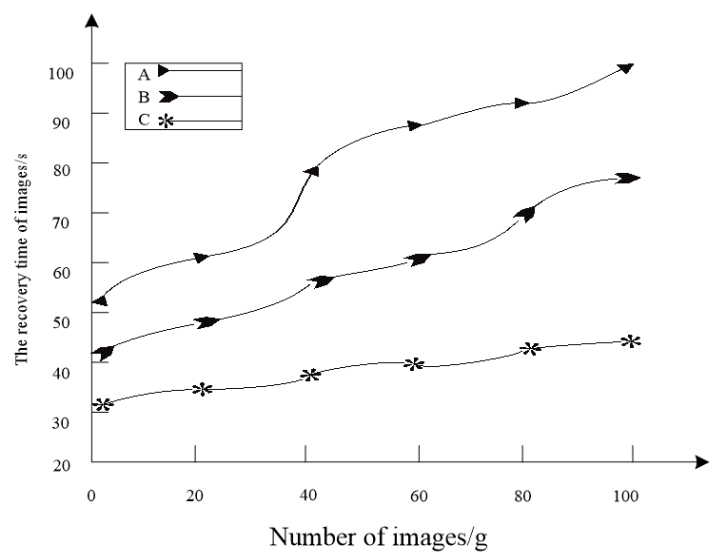

Figure 1: Computing time for the reconstruction of IEEE33 node system based on four algorithms

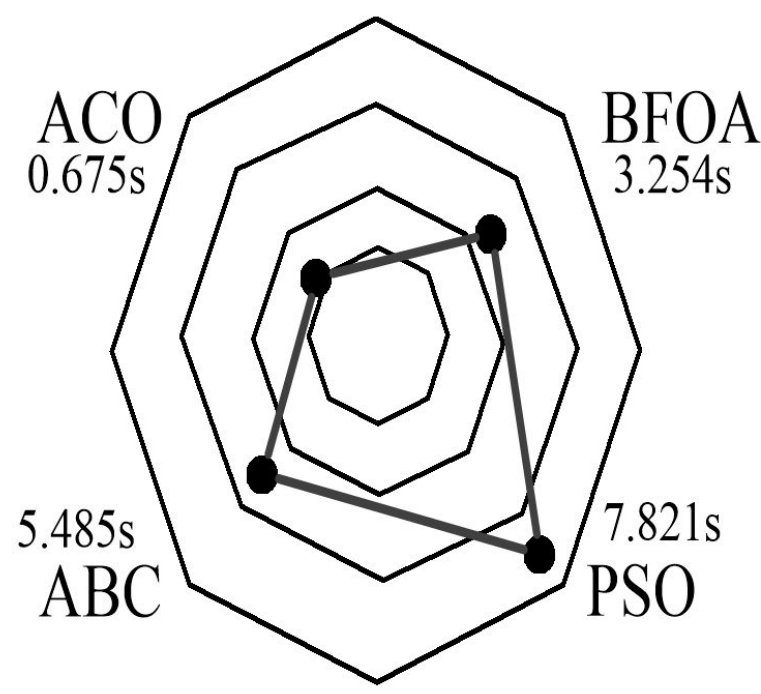

Figure 2: Computing time for the reconstruction of TaiPower84 node system based on four algorithms

at least 10 times, that of the improved bacterial foraging algorithm and the improved artificial bee colony algorithm are all 22 times, and the double-layer improved particle swarm optimization algorithm is 32 times. Table 4 also lists the number of using algorithms for the Taipower84 system calculate the power flow. The number of power flow calculations among algorithm ACO, BFOA and $\mathrm{ABC}$ is not much different, which are 20 times, 26 times and 28 times respectively. While the distribution network reconfiguration based on the double-layer improved particle swarm optimization algorithm has the largest number of calculations, which is 75 times.

Reducing network loss is the optimization goal of high voltage distribution network reconfiguration. Whether the network loss determines the accuracy and optimization performance of the reconstruction algorithm needs to prove. Figures 3 and 4 show the final network loss after reconstruction of the IEEE33 node system and the Taipower84 system based on four algorithms.

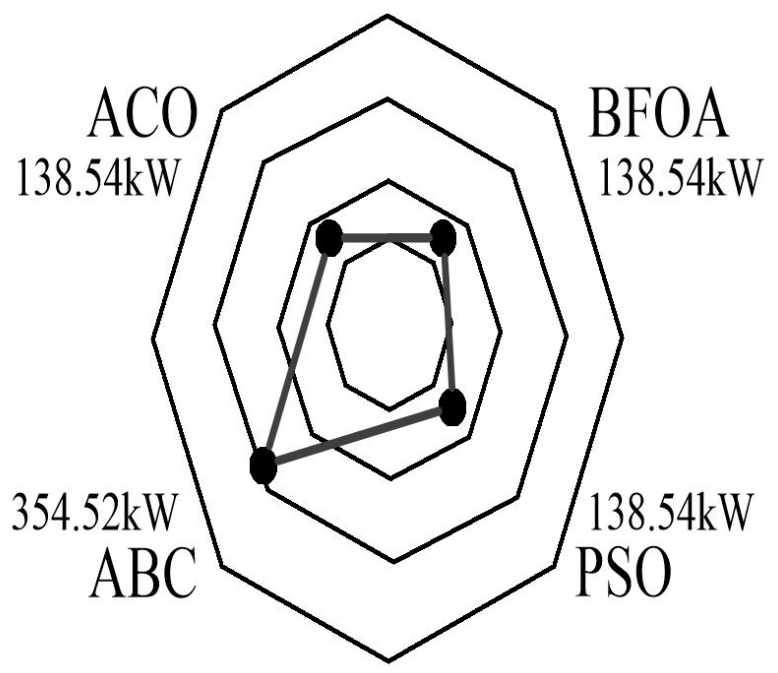

Figure 3: The final network loss after reconstruction of the IEEE33 node system based on four algorithms

It can be seen from Figure 3 that after the IEEE 33-node system is reconstructed by four algorithms, only the final network based on the improved artificial ant colony algorithm is not the global optimal solution, and the network loss is $354.52 \mathrm{~kW}$, while others three algorithms converge to the global optimal solution, and the final network loss is $138.54 \mathrm{~kW}$. 


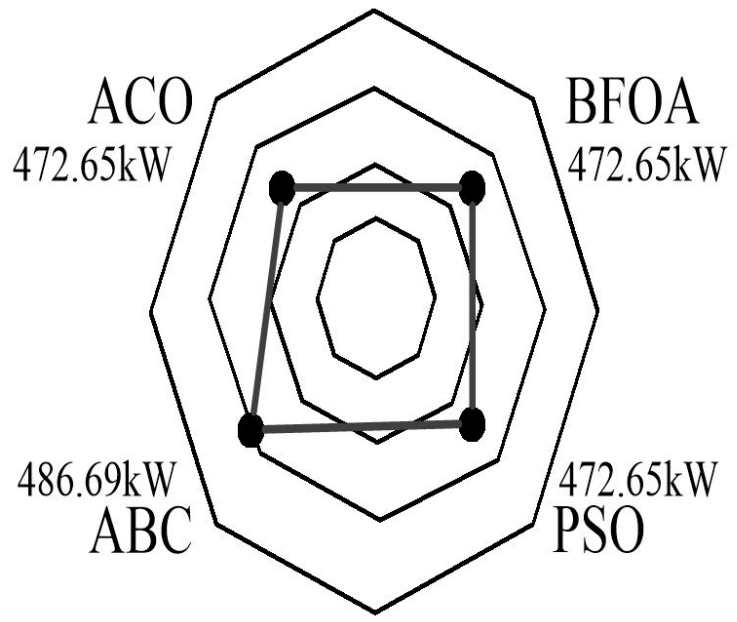

Figure 4: The final network loss after reconstruction of the Taipower84 system based on four algorithms

Figure 4 shows the final network loss obtained by using four algorithms for reconstruction calculation of the Taipower84 system. Figure 4 shows after the proposed reconstruction algorithm, the improved bacterial foraging algorithm, and the double-layer improved particle swarm optimization algorithm are applied, the Taipower84 system converges to the global optimal solution of $472.65 \mathrm{~kW}$, and the improved artificial ant colony algorithm obtains the local optimal feasible solution, and the global optimal solution is not found.

The reconfiguration of the distribution network with the goal of reducing network loss can improve the quality of the network supply voltage. Figures 5 and 6 show the minimum value of system node voltage of the IEEE33 and TaiPower84 systems after reconstruction based on four algorithms.

It can be seen from Figure 5 that the minimum value of the system node voltage is $0.9445 \mathrm{pu}$ after the reconstruction of IEEE33 node system based on the improved artificial ant colony algorithm, and the other algorithms are $0.9245 \mathrm{pu}$. For the TaiPower84 system, the overall change and the final network loss obtained by the algorithms are in the contrary. It can be seen from Figure 6 that the three algorithms that network loss is optimal after applying the reconstruction are: the proposed algorithm, the improved bacterial foraging algorithm, and the double-layer improved particle swarm optimization algorithm. The maximum network loss of the node voltage obtained by the grid reconstruction algorithm is $0.9415 \mathrm{pu}$, the minimum value of the system node voltage is $0.9515 \mathrm{pu}$ after the reconstruction of Taipower84 system based on the improved artificial ant colony algorithm, and the over-

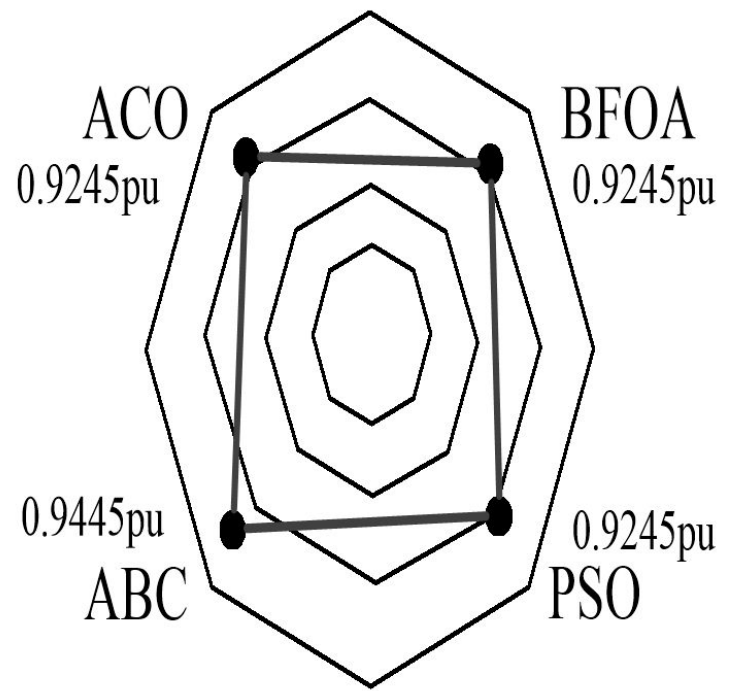

Figure 5: The minimum value of system node voltage after reconstruction of the IEEE33 system based on four algorithms

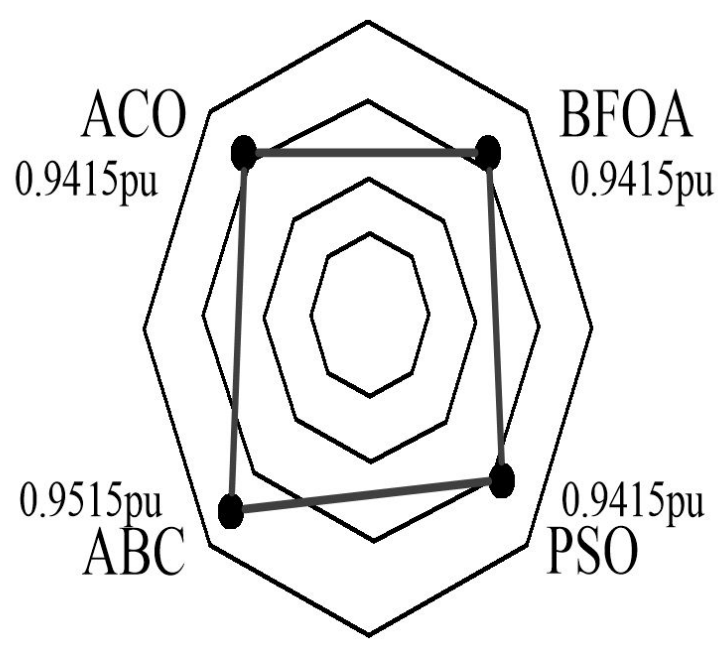

Figure 6: The minimum value of system node voltage after reconstruction of the Taipower84 system based on four algorithms

all change is opposite to the corresponding final network loss.

\section{Discussion}

In this paper, the IEEE33 node system and the TaiPower84 node system are used as examples to algorithm reconstruction time, power flow calculation number, final network loss and node voltage minimum value. It is concluded that the reconstruction algorithm based on ant colony algorithm is most suitable for solving the problem of dis- 
tribution network reconstruction; the reconstruction algorithm based on improved bacterial foraging algorithm is the second; the reconstruction algorithm based on improved artificial bee colony algorithm ranks third, and the performance of the reconstruction algorithm based on the double-layer improved particle swarm optimization algorithm is the worst. The maximum and minimum ant colony algorithm is introduced to improve the pheromone updating method of the traditional ant colony algorithm, and the search range is expanded, so that the algorithm can jump out of the local optimization trap to realize the accurate solution of the distribution network reconstruction model. The experimental results show that compared with the current network reconstruction algorithm, the proposed algorithm requires less time for convergence, less power flow calculation, and lower network loss.

\section{Conclusions}

Distribution network reconfiguration is one of the important means to ensure the safe, reliable and economic operation of the power system. From the purpose of reducing network loss, balancing system load, improving power supply reliability and voltage stability, the research on distribution network reconfiguration is deeply studied, and the goal of distribution network reconfiguration is given. The mathematical model of distribution network reconstruction problem is established by function and constraint conditions. The minimum network loss is the objective function in the model, and the topology structure of a distribution network is simplified. The power flow calculation method based on hierarchical operation mode is proposed. Based on the existing ant colony algorithm, the algorithm's improvement measures are given to form an improved ant colony algorithm. To summarize the full text, the following conclusions can be drawn:

Taking the IEEE33 node system and the TaiPower84 node system as examples, the performances of the four heuristic reconstruction algorithms are compared from the reconstruction time, power flow calculation times, final network loss and node voltage minimum value. The results show that the reconstruction algorithm based on ant colony algorithm is most suitable for solving distribution network reconstruction problem, followed by the distribution network reconstruction algorithm based on improved bacterial foraging algorithm and the reconstruction algorithm based on improved artificial bee colony algorithm, and the performance of the distribution network recon- struction algorithm based on the double-layer improved particle swarm optimization algorithm is the poorest.

Aiming at the problem of distribution network reconfiguration with the optimization of network active loss, the concept of ant colony algorithm is proposed. The performance of the proposed algorithm is tested by multiple examples. The reconstruction results show that the proposed algorithm has obvious advantages in terms of reconstruction calculation time, power flow calculation times, final network loss and node voltage's minimum value. In the future, we will improve in the following aspects:

The problem that the algorithm reconstruction depends on the initial solution is solved. The original ant colony algorithm will fail to converge to the global optimal if the initial solution is unreasonable. The application of ant colony algorithm will have a problem that the final solution depends heavily on the initial solution, which leads to that the large network reconstruction cannot search for the optimal solution.

The problem that the power flow of the distribution network cannot be efficiently and accurately calculated is solved. For the distribution network reconfiguration problem with reduced network loss as the optimization goal, the power flow calculation runs through the algorithm all the time. The efficient and accurate power flow method can ensure the reconstruction efficiency and accuracy.

\section{References}

[1] Dang C.L., Li Z.F., Dynamic Reconfiguration Optimization Strategy Based on Distribution Network Economy, Automation and Instrumentation, 2016, (4), 112-114.

[2] Deng H.C., Mao Y., Peng W.Q. et al., Distribution Network Reconfiguration Based on Improved Artificial Bee Colony Algorithm, Proceedings of the CSU-EPSA, 2016, 28(7), 125-129.

[3] Dominguez-Garcia A. D., Kassakian J.G., Schindall J. E., Reliability evaluation of the power supply of an electrical power net for safety-relevant applications, Reliability Engineering and System Safety, 2017, 91(5), 505-514.

[4] Dong X.J., Han W.J., Application of Ant Colony algorithm in Traffic Management, Journal of China Academy of Electronics and Information Technolo, 2016, 11 (6), 663-666.

[5] Dorostkar-Ghamsari M.R., Fotuhi-Firuzabad M., Lehtonen M. et al., Value of distribution network reconfiguration in presence of renewable energy resources, IEEE Transactions on Power Systems, 2016, 31(3), 1879-1888.

[6] Fletcher J.R.E., Fernando T.L., lu H. et al., Spatial optimization for the planning of sparse power distribution networks, IEEE Transactions on Power Systems, 2018, PP (99), 1-1.

[7] Ji Y., Yuan Z., Zhao J. et al., Hierarchical control strategy for mvdc distribution network under large disturbances, let Generation Transmission and Distribution, 2018, 12 (11), 2557-2565.

[8] Milani A.A., Khan M.T.A., Chakrabortty A. et al., Equilibrium 
point analysis and power sharing methods for distribution systems driven by solid-state transformers, IEEE Transactions on Power Systems, 2017, PP (99), 1-1.

[9] Peng W.Q., Mao Y., Deng H.C. et al., Application of Improved Bacterial Foraging Algorithm to Distribution Network Reconfiguration, Proceedings of the CSU-EPSA, 2017, 29 (7), 93-97.

[10] Sevlian R. A., Zhao Y., Rajagopal R. et al. Outage detection using load and line flow measurements in power distribution systems. IEEE Transactions on Power Systems, 2017, PP (99), 1-1.

[11] Wang X., Song G., Chang Z. et al., A faulty distribution network is detected based on a hybrid atomic dictionary and energy spectrum feed lines, let Generation Transmission and Distribution, 2018, 12(3), 596-606.

[12] Zang W.M., Hua H., Lai B.Y. et al., Research on Cross-platform Agile Dynamic Reconfigurable System Architecture, Journal of China Academy of Electronics and Information Technology, 2017, 12(3), 225-231.

[13] Zhou B., Littler T., Local storage meets local demand: a technical solution to future power distribution system, let Generation Transmission and Distribution, 2016, 10(3), 704-711.

[14] Zhu B., Sun Z.Z., Zhao J.Q. et al., Reconfiguration Strategy of Distribution Network with DG Based on Double Layer Improved Particle Swarm Optimization, Advances of Power System and Hydroelectric Engineering, 2017, 33(7), 39-46.
[15] Marín F., Alhama F., Solano J., Meroño P.A., Sánchez J.F., MultiScale Simulations of Dry Friction Using Network Simulation Method, Applied Mathematics and Nonlinear Sciences, 2016, 1(2), 559-580.

[16] Iqbal M., Parveen R., Parveen A., Parveen B., Aref I.M., Establishing the Botanical Identity of Plant Drugs Based On their Active Ingredients Under Diverse Growth Conditions, Journal of Environmental Biology, 2018, 39(1), 123-136.

[17] Fu H., Liu X., A Study on the Impact of Environmental Education on Individuals' Behaviors Concerning Recycled Water Reuse, Eurasia Journal of Mathematics Science and Technology Education, 2017, 13(10), 6715-6724.

[18] Peng W.X., Wang L.S., Mirzaee M., Ahmadi H., Esfahani M.J., Fremaux S., Hydrogen and Syngas Production by Catalytic Biomass Gasification, Energy Conversion and Management, 2017, 135, 270-273.

[19] Klymchuk T., Regularizing Algorithm for Mixed Matrix Pencils, Applied Mathematics and Nonlinear Sciences, 2017, 2(1), 123130.

[20] Perez Vargas J., Vigueras Carmona S.E., Zamudio Moreno E., Rivera Casado N.A., Calva Calva G., Bioremediation of Soils From Oil Spill Impacted Sites Using Bioaugmentation with Biosurfactants Producing, Native, Free-Living Nitrogen Fixing Bacteria, Revista Internacional De Contaminacion Ambiental, 2017, 33(SI), 105-114. 